\title{
ESTUDO DOS REGIMES DE ESCOAMENTO DO LEITO DE JORRO FLUIDIZADO COM TUBO DRAFT EMPREGANDO FLUIDODINÂMICA COMPUTACIONAL
}

\author{
B. S. A. ARAÚJO ${ }^{1}$, K. G. SANTOS ${ }^{1 *}$ \\ ${ }^{1}$ Universidade Federal Do Triângulo Mineiro, Departamento de Engenharia Química \\ *e-mail:kassiagsantos@gmail.com
}

\begin{abstract}
RESUMO
Os leitos de jorro fluidizado tem se mostrado promissores para operações de contato gás-sólido com e sem reações químicas, tais como secagem, revestimento, granulação, gaseificação, pirólise, entre outras. Isto decorre não somente devido ao fato de ele reunir vantagens dos leitos de jorro e fluidizado, mas principalmente devido a sua eficácia em tratar transformações químicas que envolvem simultaneamente transferência de calor e massa em combinação com partículas de diversos tamanhos. Portanto, é de fundamental importância o conhecimento detalhado da fluidodinâmica do leito, principalmente, para projetos de scale-up, o que torna a simulação computacional uma ferramenta essencial. Estudos feitos utilizando a Fluidodinâmica Computacional (CFD) mostraram-se bastante eficientes na previsão da dinâmica das partículas neste tipo de leito. Na Fluidodinâmica Computacional as duas fases são tratadas como contínuas interpenetrantes, sendo que as fases são descritas através de equações de conservação de massa, momento e energia. $\mathrm{O}$ objetivo deste trabalho foi simular empregando a técnica CFD os dados experimentais da fluidodinâmica de um leito de jorro fluidizado. Foram identificados oito regimes distintos de escoamento, os quais apresentaram-se em boa concordância com o mapa de regime apresentado por Sutkar et al. (2013). Os resultados mostraram que a técnica foi eficiente para a simulação da hidrodinâmica do leito apresentado.
\end{abstract}

\section{INTRODUÇÃO}

O leito de jorro fluidizado é um equipamento de contato fluido-partícula que reúne características de jorro e fluidização. Ele é fruto de uma série de modificações feitas nos leitos convencionais a fim de ampliar sua faixa de operacionalidade e aplicação e apresenta além da injeção de gás de jorro, distribuidores de gás de fluidização (CHATERRJEE, 1970).

Nas últimas décadas, a aplicabilidade do leito de jorro fluidizado tem crescido imensamente, o que não se deve somente ao fato de ele reunir vantagens dos leitos de jorro e fluidizado, mas principalmente devido a sua eficácia em tratar transformações físicoquímicas que envolvem simultaneamente transferência de calor e massa em combinação com partículas de diversos tamanhos. Isto é importante em aplicações tais como, granulação, revestimento, secagem, pirólise, combustão, entre outros (LINK et al, 2005; SUTKAR et al., 2013).

Apesar das inúmeras vantagens, os leitos de jorro fluidizado ainda apresentam algumas limitações quanto à operação e controle, como a instabilidade do jorro. Devido a isso, propõe-se a inserção de um tubo draft, que consiste em placas internas que irão minimizar tais limitações operacionais, uma vez que possibilita flexibilidade na altura máxima de jorro sem perturbar a estabilidade do jorro. Além disso, a inserção de um tubo draft em um leito de jorro fluidizado providencia maleabilidade 
para controlar a velocidade da partícula, a porosidade do leito e o tempo de residência da fase gasosa, ajustando parâmetros operacionais e configurações geométricas, tais como altura de arrastamento e dimensões do tubo draft (SUTKAR et al., 2013).

Ainda que seja crescente sua aplicabilidade e importância, existem poucos estudos investigando a quantificação do comportamento hidrodinâmico das partículas em um leito de jorro fluidizado com tubo draft. De acordo com a variação da vazão de alimentação de ar principal e complementar, o equipamento apresenta comportamento fluidodinâmico distinto. Desta forma, a predição por meio de simulação computacional desta fluidodinâmica pode auxiliar o projeto e otimização deste equipamento, além de auxiliar no desenvolvimento de novas aplicações e seleção de condições operacionais.

O comportamento hidrodinâmico dos leitos de jorro fluidizado não depende exclusivamente de parâmetros operacionais, tais como as velocidades de jorro e de fundo, depende também das propriedades físicoquímicas das fases envolvidas (tamanho, forma e densidade dos sólidos, bem como viscosidade, densidade e umidade do gás) e das configurações do leito, por exemplo altura do leito e diâmetro do jorro. Quaisquer alterações nesses parâmetros podem mudar drasticamente o comportamento do leito. É de extrema necessidade compreender as características do fluxo de gás e de partículas nos leitos para otimizar a operação e o projeto.

Sendo assim, é de fundamental importância a construção de mapas de regime válidos em toda a faixa operacional a fim de identificar padrões distintos de escoamento bem como regiões de transição do regime. Mapas de regime de escoamento são construídos, geralmente, através da manutenção de uma das velocidades de gás constante e alterando a outra. A velocidade de fundo é mantida em um determinado valor e a variação no padrão do fluxo é observada através do aumento gradual da velocidade de jorro. Além da observação visual, existem outros métodos que podem ser usados para construir o mapa de regime, por exemplo, flutuações de pressão, altura do leito, imagens de alta velocidade e análise de velocidades (SUTKAR; DEEN e KUIPERS, 2013).

Nagashima et al. (2015) investigaram e compararam o efeito de alguns parâmetros de operação nas características de fluxo do Leito de Jorro Fluidizado com e sem tubo draft. O estudo foi realizado utilizando a mesma coluna semi-cilíndrica e as mesmas partículas. Nagashima et al. (2015) investigaram e compararam o efeito de alguns parâmetros de operação nas características de fluxo do Leito de Jorro Fluidizado com e sem tubo draft. O estudo foi realizado utilizando a mesma coluna semi-cilíndrica e as mesmas partículas.

São muitas as técnicas utilizadas para simular sistemas gás-sólido, entre as quais se destaca a abordagem Euler-Euler. Este método, também conhecido como Fluidodinâmica computacional, adota um modelo multifásico granular em que as fases são consideradas interpenetrantes e contínuas. A técnica de Fluidodinâmica Computacional (CFD) tem sido usada como uma ferramenta numérica poderosa na modelagem de fluxos multifásicos levando à reduções de custo e tempo de projeto (HOSSEINI et al., 2009).

O objetivo do presente trabalho é estudar a fluidodinâmica de um leito de jorro fluidizado através de simulações empregando a técnica CFD. Pretende-se simular as diferentes condições operacionais, baseadas no trabalho Sutkar et al. (2013), que construiu um mapa de regimes de escoamento para o leito de jorro fluidizado com tubo draft; podendo assim contribuir para estudos na área e auxiliar no desenvolvimento de novas aplicações e otimização de processos para este equipamento. 


\section{METODOLOGIA}

\subsection{Dados experimentais de Sutkar et al. (2013)}

O trabalho experimental apresentado por Sutkar et al. (2013) foi conduzido em um leito de jorro fluidizado pseudo-2D com as seguintes dimensões: $0,14 \times 0,02 \times 1 \mathrm{~m}^{3}$. A parede frontal do leito consistia de uma placa de vidro para possibilitar a visualização do movimento das partículas no interior do leito, e a parede traseira era feita de alumínio anodizado. Além disso, foram colocadas duas placas internas dentro do leito com as seguintes dimensões: $0005 \times 0,02 \times 0,32 \mathrm{~m}^{3}$, cada uma delas localizadas a $0,045 \mathrm{~m}$ da parede lateral e $0,03 \mathrm{~m}$ acima da entrada de ar.

As partículas utilizadas eram esferas de vidro; suas propriedades estão descritas na Tabela 1.

Tabela 1 - Propriedades Físicas das partículas utilizadas.

\begin{tabular}{ccc}
\hline Propriedades & $\begin{array}{c}\text { Esfera de } \\
\text { vidro }\end{array}$ & Unidade \\
\hline $\mathbf{d}_{\mathbf{p}}$ & 1 & $\mathrm{~mm}$ \\
$\mathbf{\rho}_{\mathbf{p}}$ & 2526 & $\mathrm{Kg} / \mathrm{m}^{3}$ \\
$\mathbf{u}_{\mathbf{m f}}$ & 0,64 & $\mathrm{~m} / \mathrm{s}$ \\
$\mathbf{e}_{\mathbf{n}}$ & 0,87 & - \\
\hline
\end{tabular}

Fonte: Sutkar et al (2013).

Durante o estudo experimental foram feitas medições de pressão através de sensores localizados no lado de trás do leito, os quais foram conectados a um conversor de sinal por meio de um sistema de aquisição de dados a um computador.

Os sinais obtidos foram gravados durante quatro minutos, com uma taxa de aquisição de dados de $50 \mathrm{~Hz}$. Para cada condição de escoamento de entrada, imagens e sinais de pressão foram gravados e transformados em um domínio de amplitudefrequência.
Os experimentos foram realizados variando a velocidade de fundo de 0 a 1,75 $\mathrm{m} / \mathrm{s}$ com incremento de $0,25 \mathrm{~m} / \mathrm{s}$ e a velocidade de jorro variou de 10 a $50 \mathrm{~m} / \mathrm{s} \mathrm{com}$ incrementos de $5 \mathrm{~m} / \mathrm{s}$. Através da análise de imagem e medições de queda de pressão foram identificados oito regimes de escoamento distintos, os quais foram agrupados para formar um mapa de regime de escoamento.

Neste trabalho, serão realizadas simulações computacionais CFD que visam a reprodução das condições experimentais apresentadas por Sutkar et al. (2013), a fim de construir um mapa do regime de escoamento, de forma a variar e combinar condições de velocidade de entrada de ar principal e complementar. Cada combinação dessas variáveis, irá produzir um comportamento fluidodinâmico diferente.

\subsection{Modelagem Computacional}

As simulações CFD foram realizadas no software comercial Fluent, enquanto a malha computacional foi construída utilizando o software Gambit. Foi empregado o Modelo Euleriano Granular, que trata a fase particulada como contínua e interpenetrante, de forma que para o fechamento da modelagem são empregadas várias equações constitutivas, empregando a Teoria Cinética Granular. Este modelo é especialmente útil e computacionalmente efetivo para sistemas multifásicos densos.

O conjunto das equações de balanço de massa e quantidade de movimento juntamente com as equações constitutivas, que estão dispostas na Tabela 2, foi resolvido utilizando a técnica de volumes finitos, empregando o Algoritmo SIMPLE para estabelecer o acoplamento velocidadepressão. A malha utilizada continha inicialmente 42670 células. Após o primeiro segundo de simulação a malha foi refinada na região de entrada. 
Tabela 2 - Sistema de equações para o escoamento multifásico gás-sólido.

(1) Continuidade para a fase q:

$\frac{\partial}{\partial t}\left(\alpha_{q} \rho_{q}\right)+\nabla \cdot\left(\alpha_{q} \rho_{q} \vec{v}_{q}\right)=\sum_{p=1}^{n}\left(\dot{m}_{p q}-\dot{m}_{q p}\right)+S_{q}$

(2) Conservação de Momento para a fase contínua g:

$\frac{\partial}{\partial t}\left(\alpha_{g} \rho_{g} \vec{v}_{g}\right)+\nabla \cdot\left(\alpha_{g} \rho_{g} \vec{v}_{g} \vec{v}_{g}\right)=-\alpha_{g} \nabla p+\nabla \cdot \overline{\overline{\tau_{g}}}+\alpha_{g} \rho_{g} \vec{g}+\vec{R}_{g s}+\left(\vec{F}_{g}+\vec{F}_{l i f t, g}+\vec{F}_{v m, g}\right)$

(3) Conservação de Momento para a fase granular s (s=A, B):

$\frac{\partial}{\partial t}\left(\alpha_{s} \rho_{s} \vec{v}_{s}\right)+\nabla \cdot\left(\alpha_{s} \rho_{s} \vec{v}_{s} \vec{v}_{s}\right)=-\alpha_{s} \nabla p-\nabla p_{s}+\nabla \cdot \overline{\overline{\tau_{s}}}+\alpha_{s} \rho_{s} \vec{g}+\vec{R}_{s g}+\left(\vec{F}_{s}+\vec{F}_{l i f t, s}+\vec{F}_{v m, s}\right)$

(4) Conservação da Energia cinética para fase granular s (s=A, B)

$\frac{3}{2}\left[\frac{\partial}{\partial t}\left(\rho_{s} \alpha_{s} \theta_{s}\right)+\nabla \cdot\left(\rho_{s} \alpha_{s} \vec{v}_{s} \theta_{s}\right)\right]=\left(-p_{s} \overline{\bar{I}}+\overline{\bar{\tau}}_{s}\right): \nabla \vec{v}_{s}+\nabla \cdot\left(k_{\theta_{s}} \nabla \theta_{s}\right)-\gamma_{\theta_{s}}+\phi_{l s}$

(5) Relações constitutivas para escoamento gás-sólido:

a) Troca de energia entre as fases granular e gasosa: $\quad \phi_{g s}=-3 K_{g s} \theta_{s}$

b) O tensor tensão de Reynolds (q=g, s): $\overline{\overline{\tau_{q}}}=\alpha_{q} \mu_{q}\left(\nabla \vec{v}_{q}+\nabla \vec{v}_{q}{ }^{T}\right)+\alpha_{q}\left(\lambda_{q}-\frac{2}{3} \mu_{q}\right) \nabla \cdot \vec{v}_{q} \overline{\bar{I}}$

c) Viscosidade cisalhante da fase particulada s ( $\left.\mathrm{s}=\mathrm{s}_{1}, \mathrm{~s}_{2}\right): \quad \mu_{s}=\mu_{s, c o l}+\mu_{s, \text { cin }}+\mu_{s, f r}$

Termo colisional ( Lun et al.1984): $\quad \mu_{s, c o l}=\frac{4}{5} \alpha_{s} \rho_{s} d_{s} g_{0, s s}\left(1+e_{s s}\right)\left(\frac{\theta_{s}}{\pi}\right)^{1 / 2}$

Termo cinético (Syamlal et al. 1993): $\quad \mu_{s, c i n}=\frac{\alpha_{s} \rho_{s} d_{s} \sqrt{\theta_{s} \pi}}{6\left(3-e_{s s}\right)}\left[1+\frac{2}{5}\left(1+e_{s s}\right)\left(3 e_{s s}-1\right) \alpha_{s} g_{0, s s}\right]$

d) Viscosidade bulk (Lun et al., 1984): $\quad \lambda_{s}=\frac{4}{3} \alpha_{s} \rho_{s} d_{s} g_{0, s s}\left(1+e_{s s}\right)\left(\theta_{s} / \pi\right)^{1 / 2}$

e) Função distribuição radial (Ogawa, 1980): $g_{0, s s}=\left[1-\left(\alpha_{s} / \alpha_{s, \max }\right)^{1 / 3}\right]^{-1}$

f) Pressão de sólidos da fase granular s :

$$
p_{s}=\alpha_{s} \rho_{s} \theta_{s}+2 \rho_{s}\left(1+e_{s s}\right) \alpha_{s}^{2} g_{0, s s} \theta_{s}
$$

g) Arraste entre fases gasosa e granular, Gidaspow et al. (1992):

$$
\text { Para } \alpha_{g}>0,8 \text { (Wen e Yu, 1966): } \begin{aligned}
K_{s g} & =\frac{3}{4} C_{D}\left(\alpha_{s} \alpha_{g} \rho_{g}\left|\vec{v}_{s}-\vec{v}_{g}\right| / d_{s}\right) \alpha_{g}{ }^{-2,65} \\
C_{D} & =\left(24 / \alpha_{g} \operatorname{Re}_{s}\right)\left[1+0,15\left(\alpha_{g} \operatorname{Re}_{s}\right)^{0,687}\right]
\end{aligned}
$$

Para $\alpha_{g} \leq 0,8$ (Ergun, 1952): $\quad K_{s g}=150 \frac{\alpha_{s}\left(1-\alpha_{g}\right) \mu_{g}}{\alpha_{g} d_{s}^{2}}+1,75 \frac{\rho_{l} \alpha_{s}\left|\vec{v}_{s}-\vec{v}_{g}\right|}{d_{s}}$

h) Dissipação de energia devido colisões partícula-partícula (Lun et al., 1984) :

$\gamma_{\theta_{m}}=\left(12\left(1-e_{s s}^{2}\right) g_{0, s s} / d_{s} \sqrt{\pi}\right) \rho_{s} \alpha_{s}^{2} \alpha_{s}^{2} \theta_{s}^{3 / 2}$

i) Difusão de energia cinética (Gidaspow et al. 1992):

$$
k_{\theta_{s}}=\frac{150 d_{s} \rho_{s} \alpha_{s} \sqrt{\theta_{s} \pi}}{3844\left(1+e_{s s}\right) g_{0, s s}}\left[1+\frac{6}{5} \alpha_{s} g_{0, s s}\left(1+e_{s s}\right)\right]^{2}+2 \rho_{s} \alpha_{s}^{2} d_{s}\left(1+e_{s s}\right) g_{0, s s} \sqrt{\frac{\theta_{s}}{\pi}}
$$

Fonte: Autor (2015) 
Além das equações descritas existem algumas equações constitutivas que compõem o modelo. Para a força de arraste, utilizou-se o modelo de Gidaspow, o qual utiliza a equação de Wen e $Y u$ (1996) para $\alpha_{\mathrm{g}}>0,8$ e a equação de Ergun (1952) para $\alpha_{\mathrm{g}} \leq 0,8$. A temperatura granular para a s-ésima fase sólida é proporcional à energia cinética do movimento aleatório das partículas (SANTOS et al., 2009) e é descrita pela Equação 04. A dissipação de energia devido às colisões entre as partículas é contabilizada pela equação derivada de Lun et al. (1984).

\subsection{Simulações CFD do leito de jorro fluidizado com tubo draft.}

A Figura 1 apresenta o mapa de regime com as condições abordadas para o leito de jorro fluidizado, incluindo os oito regimes simulados (pontos em preto). As condições de velocidade estudadas e simuladas neste trabalho estão explícitas na Tabela 3. Os regimes estudados foram: S1 - Leito Fixo; S2 - Jorro Intermitente; S3 - Jorro com Aeração (Jorro Agregado); S4 - Jorro com Aeração; S5 - Jorro Fluidizado com Aeração (Disperso); S6 - Jorro Fluidizado com Aeração (Agregado); S7 - Fluidizado com Jorro Slugging; S8 - Leito Fluidizado, como mostra a Tabela 3.

Tabela 3 - Condições estudadas nas Simulações CFD dos 8 diferentes regimes de escoamento.

\begin{tabular}{ccccc}
\hline Simulação & $\mathbf{U}_{\mathbf{b g}} / \mathbf{U}_{\mathbf{m f}}$ & $\mathbf{U}_{\mathbf{s p}} / \mathbf{U}_{\mathbf{m f}}$ & $\begin{array}{c}\mathbf{U}_{\mathbf{b g}} \\
{[\mathbf{m} / \mathbf{s}]}\end{array}$ & $\begin{array}{c}\mathbf{U}_{\mathbf{s p}} \\
{[\mathbf{m} / \mathbf{s}]}\end{array}$ \\
\hline S 1 & 0,28 & 0,00 & 0,18 & 0,00 \\
S 2 & 0,28 & 20,00 & 0,18 & 12,80 \\
S 3 & 0,50 & 42,00 & 0,32 & 26,88 \\
S 4 & 0,60 & 60,00 & 0,38 & 38,40 \\
S 5 & 2,00 & 60,00 & 1,28 & 38,40 \\
S 6 & 2,00 & 32,00 & 1,28 & 20,48 \\
S 7 & 2,00 & 18,00 & 1,28 & 11,52 \\
S 8 & 2,00 & 2,00 & 1,28 & 1,28 \\
\hline
\end{tabular}

Fonte: Autor (2015).

Figura 1 - Mapa de regime de regimes de escoamento do leito de jorro fluidizado: • condições simuladas.

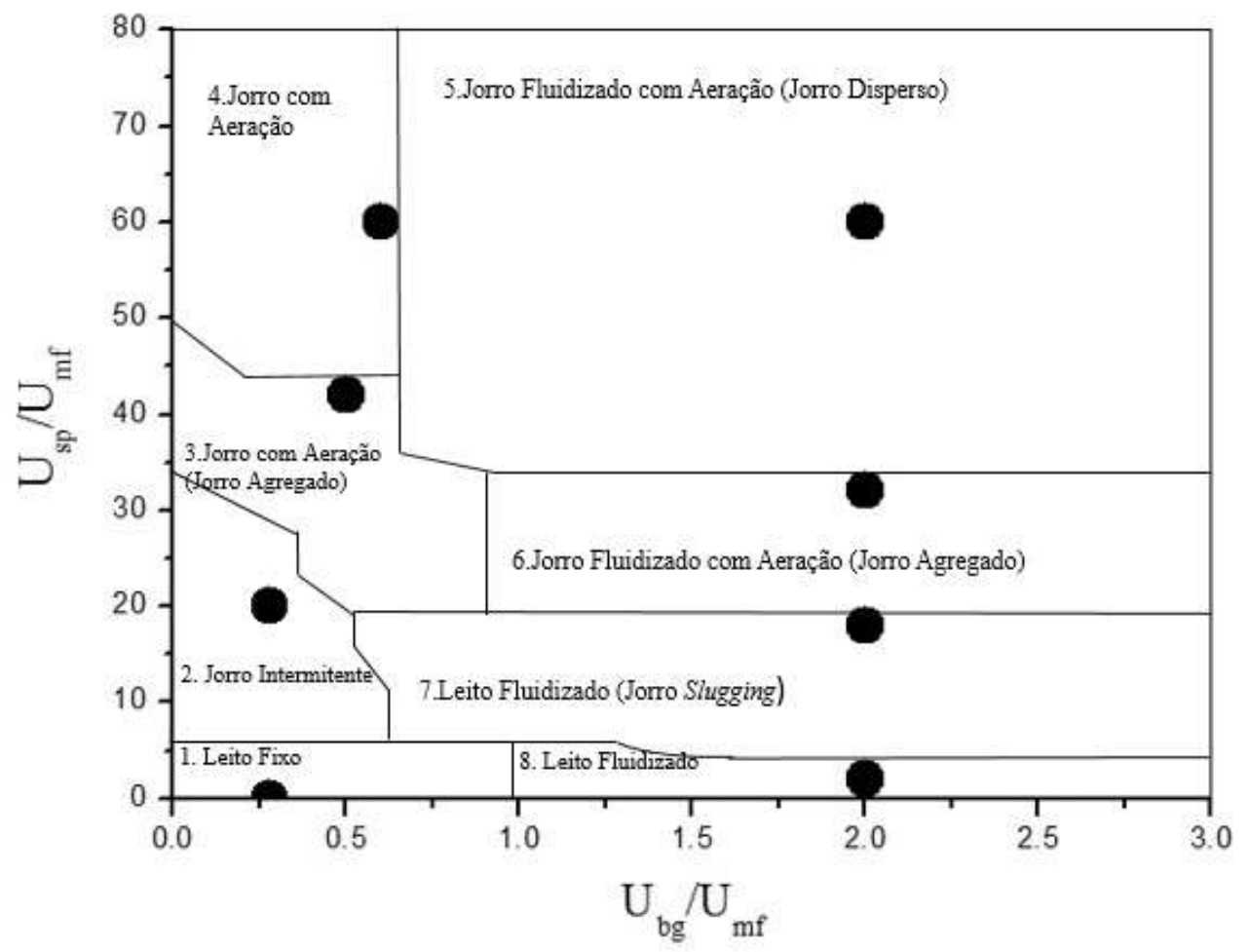

Fonte: Autor (2015). 


\section{RESULTADOS}

Analisando a Figura 4, nota-se que a simulação 1 apresenta um regime de leito fixo, o qual apresenta-se a baixas velocidades de jorro e de fundo e onde as partículas não se movimentam, enquanto isso na simulação 2 referente ao jorro intermitente, são observados deslocamentos consideráveis das partículas entre os tubos draft e a formação de bolhas próximo à placa inferior com o aumento da velocidade de jorro. Nota-se que as partículas na região anular permanecem estáticas, o que ocorre devido à baixa velocidade de fundo. Além disso, as partículas não são continuamente transportadas do jorro para a região anular como pode ser melhor visualizado nos perfis de velocidade e fração volumétricas apresentados.

Pode-se perceber pela simulação 3, que diz respeito ao Jorro com Aeração (sendo o jorro agregado), que este regime apresenta a formação de um jorro estável no qual as partículas são continuamente transportadas através do tubo draft, sendo que ao atingi-lo elas perdem velocidade e fluem para baixo através da região anular, formando algo semelhante a uma fonte. Esse mesmo comportamento foi observado por Sutkar et al. (2013) ao realizar seu trabalho experimental sob condições semelhantes às simuladas. Foi observado também a formação de clusters na superfície interna dos tubos.

Para a simulação 4, que se refere ao Jorro com Aeração, pode-se observar que para altas velocidades de jorro as partículas são lançadas através do tubo draft, de forma totalmente dispersa e sem formação de clusters.

Comparando-o ao regime anterior, nota-se que no jorro as partículas aqui são elevadas a alturas maiores mostrando uma distribuição homogênea de partículas. Com isso, tem-se a formação de uma carga circulante de partículas, com altas velocidades no jorro e baixas velocidades na região anular. Segundo Sutkar et al. (2013) este tipo de regime é ideal para aplicações que requerem altas taxas de transferência de calor e massa e uma mistura de partículas somente no jorro.

Figura 4 - Contorno da fração volumétrica de sólidos: regimes de escoamento do Leito de Jorro Fluidizado.

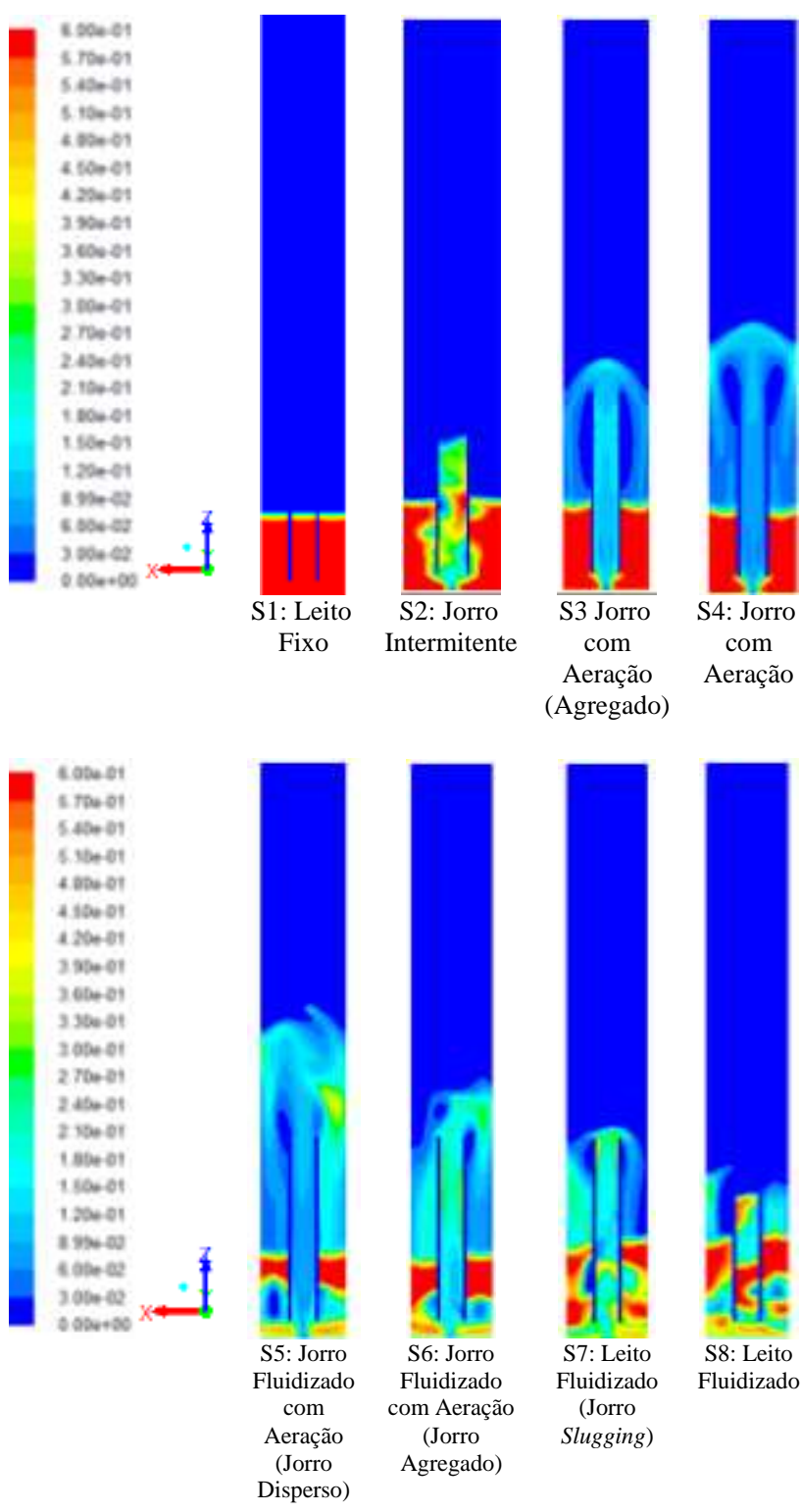

Fonte: Autor (2015)

Ao avaliar a simulação 5, a qual se refere ao Jorro Fluidizado com Aeração (Jorro Disperso), pode-se notar que as partículas são elevadas através do tubo draft em um estado 
de total dispersão, o que pode ser justificado pelas altas velocidades de fundo e de jorro utilizadas nesta condição. Além disso, isso leva a um movimento intenso de partículas na região anular ocasionando a formação de bolhas, como ser observado no perfil de fração volumétrica.

A diferença deste regime em relação aos regimes de Jorro Fluidizado com Aeração (Jorro Agregado) e Jorro com Aeração, citado anteriormente, está no transporte contínuo de partículas que alcança altas alturas de leito com a região anular fluidizada.

A simulação 6 referente ao Jorro Fluidizado com Aeração (Jorro Agregado) tem as partículas continuamente transportadas da região anular para o fundo do leito, além de apresentar uma formação caótica de bolhas na região, como visto na Figura 4.

$\mathrm{Na}$ Simulação 7 tem-se um Leito Fluidizado com Jorro Slugging, onde pode-se observar um deslocamento intenso de partículas nas regiões de jorro e anular, com formação de bolhas e golfadas (slugging). No jorro, observa-se uma frequente geração de bolhas próximo às placas de distribuição, as quais irão criar uma maior resistência ao gás de jorro, bloqueando parcialmente o tubo draft.

Devido à pequena distância entre os tubos draft pode-se observar que há a formação de bolhas alongadas verticalmente, o que resulta numa súbita erupção de partículas, que também pode ser devido à velocidade de jorro ser muito baixa e não ser capaz de transportar as partículas através do tubo draft.

A simulação 8 apresenta um Leito Fluidizado, no qual as partículas se movimentam tanto na região de jorro quanto na região anular, devido à velocidade de fundo ser maior que a velocidade de mínima fluidização, principalmente. Com o aumento das velocidades de fundo e de jorro, as partículas são levantadas pela formação de bolhas no jorro ou na região anular.

Ao analisar a Figura 4 nota-se a intensa movimentação de partículas na região anular, que é explicada devido a formação de bolhas.

A Figura 5 traz a trajetória das partículas na entrada, saída e no interior do tubo draft, para o regime de jorro fluidizado com aeração: com jorro agregativo (a) e disperso (b). Observa-se que o formato da fonte tende a ser cônico no jorro agregativo, enquanto no disperso a fonte não é simétrica.

Figura 5 - Vetor velocidade das partículas de vidro para a condição de jorro fluidizado com aeração: (a) jorro agregativo (Simulação 6); (b) Jorro disperso (simulação 5).
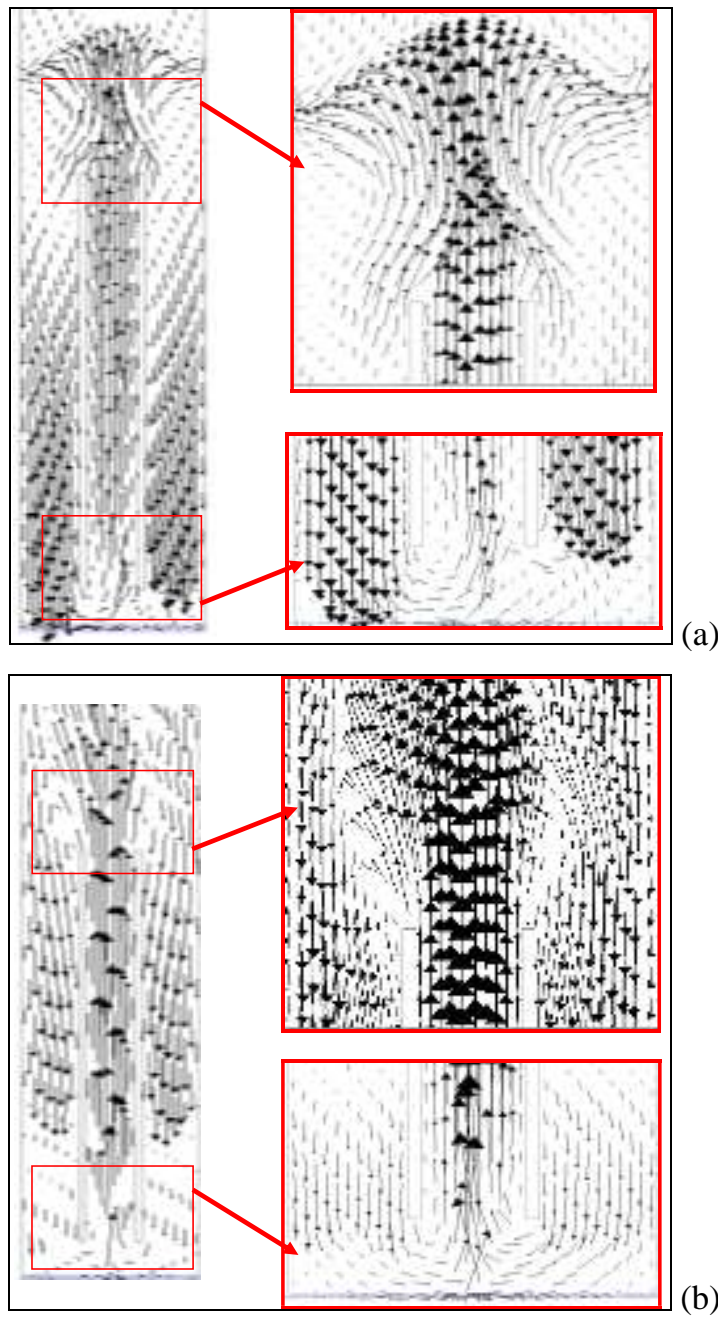
A Figura de 6 mostra uma comparação entre as velocidades das partículas ao longo da direção radial em diversas alturas para os regimes de jorro com aeração (jorro agregado), jorro com aeração e jorro fluidizado com aeração (jorro disperso) das simulações obtidas neste trabalho $\mathrm{e}$ as simuladas por Sutkar et al. (2013). Nota-se que os comportamentos simulados são qualitativamente similares aos investigados por Sutkar et al. (2013).

Para o regime de jorro com aeração (jorro agregado), Figura 5, para a altura de 10 $\mathrm{cm}$ observa-se que as partículas tem maiores velocidades no jorro, enquanto que para os outros dois regimes, as partículas tem altas velocidades nas regiões anular e de jorro.

Figura 6 - Comparação qualitativa entre os resultados de Sutkar et al. (2013) e os simulados: velocidade das partículas na direção z para o regimes de: (a) Jorro com aeração agregada (simulação 3); (b) Regime de jorro com aeração (simulação 4); (c) regime de jorro fluidizado com aeração dispersa (simulação 5).
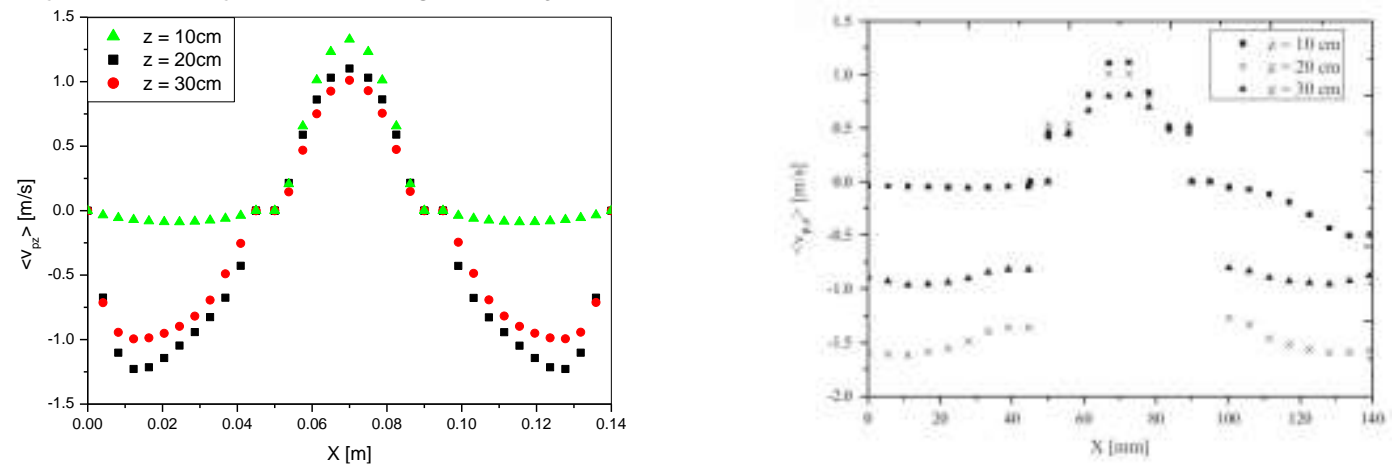

(a) Regime de Jorro com aeração agregada
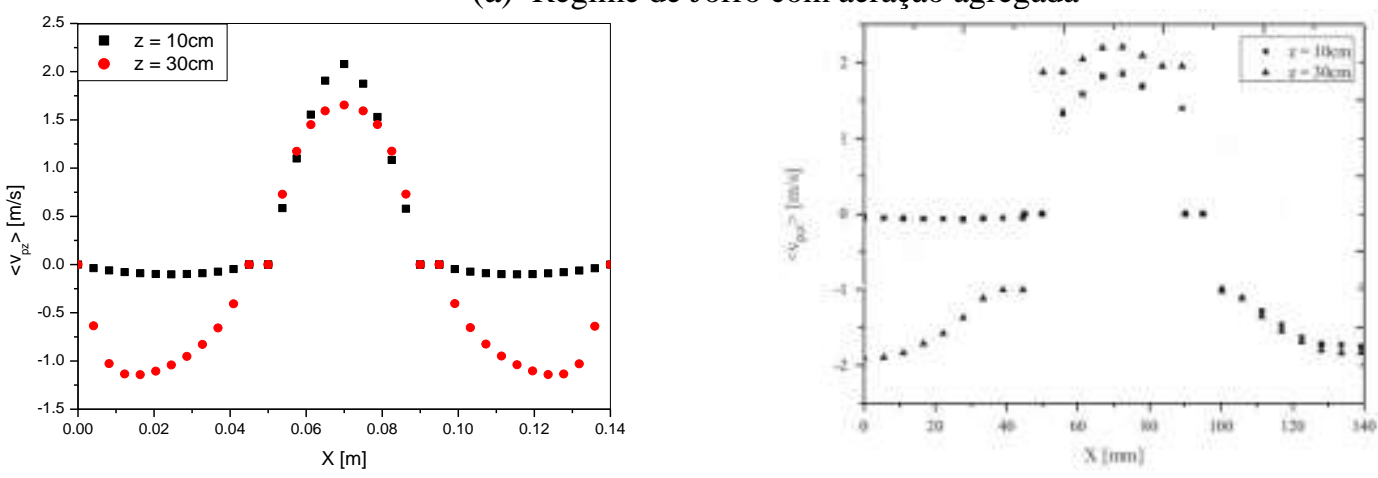

(b) Regime de jorro com aeração
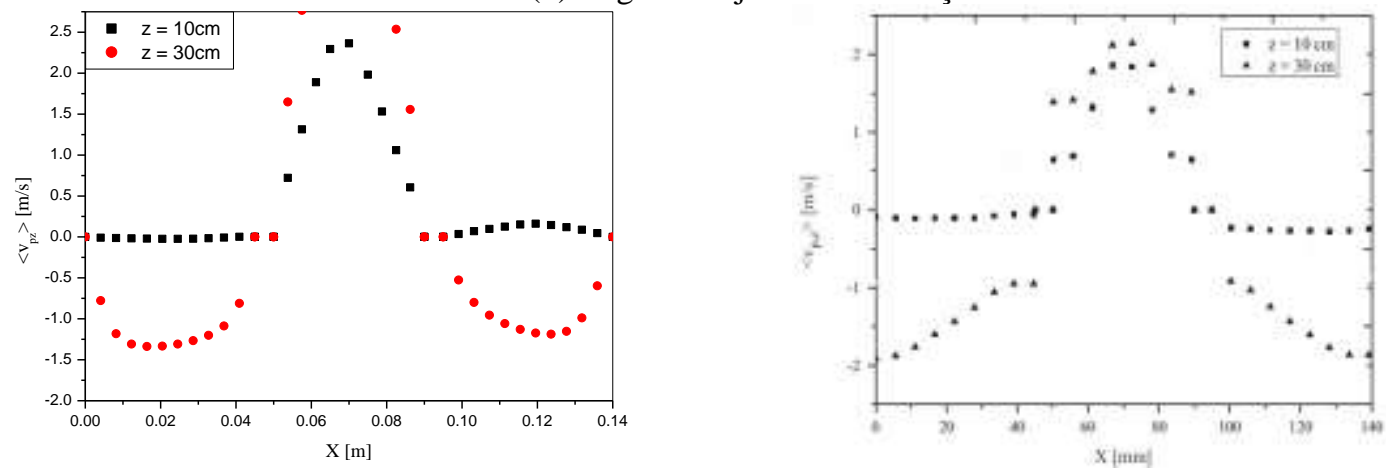

(b) Regime de jorro fluidizado com aeração dispersa

Fonte: Do Autor (2015) 


\section{CONCLUSÃO}

Neste estudo, foram investigados oito regimes distintos, mudando a velocidade de fundo e de jorro. Nota-se que com o aumento da velocidade de jorro, o movimento das partículas no leito aumenta para toda a faixa de velocidade de fundo. Em velocidades de jorro e de fundo baixas, observa-se que quase não há deslocamentos de partículas na região de jorro e anular. Entretanto, foi observado um transporte contínuo de partículas e altas taxas de circulação através da região anular fluidizada, à medida que as velocidades aumentam.

Os resultados encontrados foram condizentes com os apresentados por Sutkar et al. (2013) em seu trabalho experimental. Além disso, os resultados mostram que a técnica foi eficiente para a simulação da hidrodinâmica do leito de jorro fluidizado com tubo draft.

\section{NOMENCLATURA}

\begin{tabular}{ll}
$\mu_{g}$ & Viscosidade cisalhante \\
$\lambda_{g}$ & Viscosidade bulk da fase gasosa \\
$F_{g}$ & Força de corpo externa \\
$\vec{v}_{g}$ & Velocidade na interface \\
$\mu_{s}$ & Viscosidade cisalhante granular \\
$\lambda_{s}$ & Viscosidade bulk granular \\
$\vec{v}_{s g}$ & Velocidade na interface \\
$\bar{\tau}_{g}$ & Tensor tensão da fase gasosa \\
\hline $\bar{\tau}_{s}$ & Tensor tensão de sólido \\
$\mu_{s}$, cin & Viscosidade cinética \\
$\mu_{s}$, col & Viscosidade devido a colisão das \\
$C_{d}$ & partículas \\
$C_{f r, s a}$ & Coeficiente de arraste \\
& Coeficiente de fricção entre as \\
$d_{p}$ & partículas da fase sólida s e a \\
& Diâmetro da partícula
\end{tabular}

\begin{tabular}{|c|c|}
\hline$d s$ & Diâmetro da partícula da fase s \\
\hline$g_{0, s s,} g_{0, a s}$ & Função distribuição radial \\
\hline$K_{s g}$ & $\begin{array}{l}\text { Coeficiente de troca de momento } \\
\text { entre as fases }\end{array}$ \\
\hline$\dot{m}$ & Taxa de transferência de massa \\
\hline$p$ & Pressão compartilhada pelas fases \\
\hline$R e$ & Número de Reynolds \\
\hline$R_{s g}$ & $\begin{array}{l}\text { Força de interação entre as fases } \\
\text { gasosa e sólida }\end{array}$ \\
\hline$S_{g}, S_{s}$ & $\begin{array}{l}\text { Termo de geração da propriedade } \\
\text { na equação de conservação }\end{array}$ \\
\hline$t$ & Tempo \\
\hline$u$ & Velocidade \\
\hline$v g$ & Velocidade do gás \\
\hline$\alpha_{g}$ & Fração volumétrica da fase gasosa \\
\hline$\alpha_{s}$ & $\begin{array}{l}\text { Fração volumétrica da fase } \\
\text { granular }\end{array}$ \\
\hline$\theta_{s}$ & Temperatura Granular \\
\hline$\rho_{g}$ & Densidade da fase gasosa \\
\hline$\rho_{s}$ & Densidade da fase granular \\
\hline$k \theta s$ & $\begin{array}{l}\text { Coeficiente de difusão de } \\
\text { temperatura granular }\end{array}$ \\
\hline$e_{s s}, e_{s a}$ & $\begin{array}{l}\text { Coeficiente de restituição entre } \\
\text { sólidos }\end{array}$ \\
\hline
\end{tabular}

$d_{a} \quad$ Diâmetro da partícula da fase a

\section{REFERÊNCIAS}

CHATTERJEE, A. Spout-fluid bed technique. Ind. Eng. Chem. Process Des. Dev., v. 9, p.340-341, 1970.

ERGUN, S. Fluid flow throug packed columns. Chemical Engineering Progress. v. 48, p. $89-94,1952$.

GIDASPOW, D.; BEZBARUAH R.; DING, J. Hydrodynamics of Circulating Fluidized Beds: Kinetic Theory Approach, In: Proc. of the 7th Engineering Foundation Conference on Fluidization, 1992. p. 75-82. 
HOSSEINI, S. H.; ZIVDAN, M.; RAHIMI, R. CFD simulation of gas-solid flow in a spouted bed with a non-porous draft tube. Chemical Engineering and Processing: Process Intensification. v. 48, 1539-1548, 2009.

LINK, J.M.; CUYPERS, L.A.; DEEN, N.G.; KUIPERS, J.A.M. Flow regimes in a spoutfluid bed: A combined experimental and simulation study. Chemical Engineering Science, v. 60, p. 3425-3442, 2005.

LUN, C.K.K.; SAVAGE, S. B.; JEFFREY,D. J.; CHEPURNIY, N. Kinetic theories for granular flow: Inelastic particles in couette flow and slightly particles in a general flow field. J. Fluid Mech, v. 140, p.223-256, 1984.

NAGASHIMA, H.; KAWASHIRI, Y.; SUZUKAWA, K.; ISHIKURA, T. Effects of Operating Parameters on Hydrodynamic Behavior of Spout-Fluid Beds without and with a Draft Tube. Procedia Engineering, v. 102, 952-958, 2015.

OGAWA, S.; UMEMURA, A.; OSHIMA, N. On the Equation of Fully Fluidized Granular Materials. J. Appl. Math. Phys.,v. 31, p. 483, 1980.

SANTOS, K. G.; MURATA, V. V.; BARROZO, M. A. S. Three-dimensional computational fluid dynamics modeling of spouted bed, Canadian Journal of Chemical Engineering, v. 87, p. 211-219, 2009.

SUTKAR, V. S.; DEEN, N.G.; KUIPERS, J.A.M. Spout Fluidized Beds: Recent advances in experimental and numerical studies. Chemical Engineering Science. v. 86, p. 124-136, 2013.

SUTKAR, V. S.; VAN HUNSEL, T.J.K.; DEEN, N. G.; SALIKOV, V.; STEFAN HEINRICH, S. A.; KUIPERS, J.A.M. Experimental Investigations of
Hydrodynamics of a Spout Fluidized Bed with Draft Plates. In: The 14th International Conference on Fluidization - From Fundamentals to Products, Holanda, 2013.

SYAMLAL, M. The particle-particle drag term in a multiparticle model of fluidization. National Technical Information Service, Springfield, VA, 1987.

WEN, C.Y; YU, Y. H. Mechanics of Fluidization. Chem. Eng. Prog. Symp. Series, v. 62, p.100-111, 1966. 